\title{
A Practical (Non-interactive) Publicly Verifiable Secret Sharing Scheme
}

\author{
Mahabir Prasad Jhanwar \\ C R RAO AIMSCS \\ University of Hyderabad Campus \\ Hyderabad, India \\ mahavir.jhawar@gmail.com
}

\begin{abstract}
A publicly verifiable secret sharing (PVSS) scheme, proposed by Stadler in [Sta96], is a VSS scheme in which anyone, not only the shareholders, can verify that the secret shares are correctly distributed. PVSS can play essential roles in the systems using VSS. Achieving simultaneously the following two features for PVSS is a challenging job:

- Efficient non-interactive public verification.

- Proving security for the public verifiability in the standard model. In this paper we propose a $(t, n)$-threshold PVSS scheme which satisfies both of these properties. Efficiency of the non-interactive public verification step of the proposed scheme is optimal (in terms of computations of bilinear maps (pairing)) while comparing with the earlier solution by [HV08]. In public verification step of [HV08], one needs to compute $2 n$ many pairings, where $n$ is the number of shareholders, whereas in our scheme the number of pairing computations is 4 only. This count is irrespective of the number of shareholders. We also provide a formal proof for the semantic security (IND) of our scheme based on the hardness of a problem that we call the $(n, t)$-multi-sequence of exponents Diffie-Hellman problem (MSE-DDH). This problem falls under the general Diffie-Hellman exponent problem framework [BBG05].

Keywords: Secret sharing, non-interactive PVSS, general Diffie-Hellman exponent problem.
\end{abstract}

\section{Introduction}

(Verifiable) Secret Sharing is one of the most important tools in modern cryptography. The concept and the first realization of secret sharing were presented independently in [Sha79] and in [Bla79]. Since then much work has been put into the investigation of such schemes (see [Sim88,Sti92] for a list of references). In a secret sharing scheme, there exists a dealer and $n$ shareholders (sometimes referred to participants). The dealer splits a secret, say $s$, into $n$ different pieces, called shares, and sends one share to each shareholder. An access structure describes which subsets of shareholders are qualified to recover the secret. By a $(t, n)$-threshold access structure, $1 \leq t \leq n$, we means that any subset of $t$ or more shareholders will be able to recover the secret; any smaller subset of shareholders will not be able to gain any information about the secret. 
The verifiable secret sharing (VSS) schemes constitute a particular interesting class of schemes as they allow each receiver of information about the secret (share of the secret) to verify that the share is consistent with the other shares. If the dealer trusts one of the shareholders completely, he could share the 'whole' secret with the person and thus altogether avoid the trouble of using a secret sharing scheme. Therefore in many applications the dealer doesn't trust the shareholders completely, and therefore it is reasonable to expect that (some of) the shareholders do not trust the dealer either. For this reason efficient verifiable secret sharing schemes are necessary in practice. Verifiable secret sharing was proposed first in [CGMA85]. In a VSS scheme, the shareholders can verify the validity of their shares and thus overcome the problem of dishonest dealers. VSS is known to play important roles in various cryptographic protocols such as the multiparty protocols [CCD88,BOGW88], key-escrow cryptosystems [Mic92], and threshold cryptography. A VSS scheme is called non-interactive if the shareholders can verify their share without talking to each other or the dealer. Proposals by [Fel87,Ped91] contributed to non-interactiveness and improved efficiency.

(Non-interactive) Publicly Verifiable Secret Sharing: The first proposed VSS scheme [CGMA85] has the special property that anyone, not only the shareholders, can verify that the shares were correctly distributed. In [Sta96], the property was named public verifiability and the VSS schemes with the above property were named publicly verifiable secret sharing schemes (PVSS). Some of the important PVSS schemes were presented in [Sta96,FO98,Sch99].

In most PVSS schemes, the verification procedure involves interactive proofs of knowledge. These proofs are made non-interactive by means of the Fiat-Shamir technique [FS86] and thus security for verifiability can only be carried out in the random oracle model [BR93]. Transforming security analysis of cryptographic primitives from the framework of random oracle model to the standard model have always turned out to be a theoretically important task which is seemingly difficult in most of the cases. Some of these problems were dealt in [RV05,HV08]. Some of the positive features of [HV08] are: non-interactive PVSS, Fiat-Shamir technique is not used, unconditional security for public verifiability and security for indistinguishability of secrets. The scheme [HV08] is described briefly in Appendix B.

Although, [HV08] successfully avoids Fiat-Shamir technique, their public verification algorithm is inefficient. In particular, for $n$ shareholders, one has to compute $2 n$ many pairings in the public verification algorithm. This number of pairing computations is expensive. Therefore, an important problem was to reduce the number of pairing computations during the public verification algorithm.

Our Contribution: In this paper we propose a practical and provably secure non-interactive $(t, n)$-threshold PVSS scheme. Our scheme achieves the following:

- Public verification algorithm is non-interactive and is obtained without using Fiat-Shamir zero knowledge proofs. 
- Comparing with the public verification step of [HV08], our scheme provides optimal efficiency in terms of the number of pairing computations. In public verification step of [HV08], one needs to compute $2 n$ many pairings, where $n$ is the number of shareholders, whereas in our scheme the number of pairing computations is 4 only. This count is irrespective of the number of shareholders. We also observe [Rev] that a simple modification to the verification algorithm of [HV08] reduces the number of pairing computations from $2 n$ to $n+1$. But this modification is done at the cost ([HV08] enjoys unconditional security for public verifiability) of reducing the security of public verifiability to a new computational problem. See Appendix C for the modified scheme and the security analysis for public verifiability.

- The scheme is provably secure against a SA-IND (see Section 2.2) adversary. The security relies on the hardness of a problem that we call the $(n, t)$ - multisequence of exponents Diffie-Hellman problem (MSE-DDH). This problem falls under the general Diffie-Hellman exponent problem framework [BBG05].

Overview of the paper: We define the syntactics of non-interactive $(t, n)$ threshold publicly verifiable secret sharing (PVSS) scheme and the required security properties in Section 2, where we also describe the $(n, t)$-MSE-DDH problem, on which the security of our scheme will be based. In this section we also recall the definition of bilinear maps. In Section 3, we describe our new scheme and discuss some of the issues. Section 3 ends with a formal security proof of our scheme. The work is concluded in Section 5 .

\section{Preliminaries}

In this section we describe the algorithms that form a non-interactive $(t, n)$ threshold publicly verifiable secret sharing (PVSS) scheme, as well as the basic security requirements for such schemes. We also introduce the computational problem called the $(n, t)$-MSE-DDH problem, to which we will relate the security of our scheme.

\section{$2.1 \quad$ (Non-interactive) PVSS}

In this section we describe a model for non-interactive PVSS. In a PVSS scheme, a dealer $D$ wishes to distribute shares of a secret value " $s$ " among $n$ shareholders $P_{1}, \ldots, P_{n}$. An access structure describes which subsets of shareholders are qualified to recover the secret. In this article, we consider $(t, n)$-threshold access structure, $1 \leq t \leq n$, which means that any subset of $t$ or more shareholders will be able to recover the secret; any smaller subset will not be able to gain any information about the secret, unless a computational assumption is broken. A PVSS scheme is described by the following standard algorithms.

- Initialization This algorithm generates all system parameters. Furthermore, each shareholder $P_{i}$ registers its public-key (may be issued by the dealer with the corresponding secret key). The actual set of shareholders 
taking part in a run of PVSS scheme must be a subset of the registered shareholders. We assume w.l.o.g. that shareholders $P_{1}, \ldots, P_{n}$ are the actual shareholders in the run described below.

- Distribution The distribution of the shares of a secret " $s$ " is performed by the dealer $D$. The dealer computes and publishes the secret commitment value(s) and the share deriving value(s) respectively. The secret commitment value(s) commits the dealer to the value of secret $s$, whereas the share deriving value(s) can be used with the shareholders' secret keys to yield the share of the secret for the respective shareholders.

- Verification It is required that the dealer's commitment to the secret can be verified publicly. Thus any party knowing only the publicly available information may verify that share deriving information is consistent with the share commitment information, i.e., it guarantees that the reconstruction protocol will be able to recover the same secret $s$. Furthermore, this verification runs non-interactively.

- Reconstruction The shareholders construct their shares $S_{i}$ from the share deriving value using the secret keys. It is not required that all shareholders succeed in doing so, as long as a qualified set of shareholders is successful. These shareholders then release $S_{i}$ and also the share commitment value(s) to verify that the released shares are correct. The share commitment information is used to exclude the shareholders which are dishonest or fail to reproduce their share $S_{i}$ correctly. Reconstruction of the secret $s$ can be done from the shares of any qualified set of shareholders.

In non-interactive PVSS schemes it is essential that all commitments can be verified non-interactively. Since any party can verify the output of the dealer, so we don't budget operations for the individual participants to check their own shares. Hence it suffices to have just one public verifier.

\subsection{Security Model}

Such a scheme must satisfy the following properties.

- Correctness: If the dealer and the shareholders act honestly, every qualified subset of shareholders reconstructs the secret during the reconstruction algorithm.

- Verifiability: If a dealer passes the verification step, then it implies that the secret commitment values are consistent with the share deriving values, i.e., the information which the dealer outputs for shareholders to derive their respective shares of the secret for which the dealer had published his commitment in terms of secret commitment values.

- Privacy: The very basic requirement is that, for an honest dealer, the adversary cannot learn any information about the secret at the end of the protocol.

Privacy: Following [RV05,HV08], we can more formally define the above privacy notion, under the classical semantic-security notion [GM84], using a game 
between an adversary $\mathcal{A}$ and a challenger. The adversary here is a static one i.e., at the beginning of the game, he is given the secret keys of the corrupted shareholders.

Indistinguishability of Secrets (IND): The security notion is defined via the following game between a challenger and a probabilistic polynomial time (PPT) adversary $\mathcal{A}$. Both the adversary and the challenger are given as input a security parameter $\lambda$.

- Initialization: The challenger runs Initialization $(\lambda)$ to obtain the set of public parameters along with the public keys and the secret keys of all the shareholders. Besides all the public keys, the adversary is also given the secret keys of $t-1$ corrupted shareholders.

- Challenge: The challenger picks two random secrets $T_{0}$ and $T_{1}$ and a random bit $b \in\{0,1\}$. Then he runs the distribution algorithm for the secret $T_{b}$ and sends all the resulting information to $\mathcal{A}$ along with $\left\{T_{0}, T_{1}\right\}$.

- Guess: Finally, the adversary $\mathcal{A}$ outputs a guess bit $b^{\prime} \in\{0,1\}$ for $b$ and wins the game if $b^{\prime}=b$.

We define the advantage of this static adversary (SA), $\mathcal{A}$, against a $(t, n)$ threshold PVSS as follows:

$$
\operatorname{Adv}_{P V S S, \mathcal{A}}^{S A-I N D}(\lambda)=\left|\operatorname{Prob}\left[b^{\prime}=b\right]-\frac{1}{2}\right|
$$

The advantage is a function of the security parameter $\lambda$.

Definition 1. We say that a $(t, n)$-threshold PVSS scheme is SA-IND secure if for all PPT adversaries $\mathcal{A}$, we have that $A d v_{P V S S, \mathcal{A}}^{S A-I N D}(\lambda)$ is a negligible function in $\lambda$.

\subsection{Bilinear Map}

Let $G_{1}, G_{2}$ and $\tilde{G}$ be three cyclic groups of prime order $p$. The group laws for all the three groups are noted multiplicatively. A mapping $e: G_{1} \times G_{2} \rightarrow \tilde{G}$ is called an admissible bilinear map (pairing) if it satisfies the following properties:

- Bilinearity: $e\left(g_{1}^{\alpha}, g_{2}^{\beta}\right)=e\left(g_{1}, g_{2}\right)^{\alpha \beta}$ for all $g_{1} \in G_{1}, g_{2} \in G_{2}$ and $\alpha, \beta \in \mathbb{Z}_{p}$.

- Non-degeneracy: $e\left(g_{1}, g_{2}\right) \neq 1$ unless $g_{1}=1$ or $g_{2}=1$.

- Computability: There exist efficient algorithms to compute the group operations in $G_{1}, G_{2}, \tilde{G}$ as well as the map $e(\cdot, \cdot)$.

A bilinear map group system is a tuple $\left(p, G_{1}, G_{2}, \tilde{G}, e(\cdot, \cdot)\right)$ composed of the objects as described above. The above bilinear map is defined in asymmetric setting [BLS01,BW06]. Also in asymmetric setting, existence of an efficiently computable isomorphism $\phi: G_{2} \rightarrow G_{1}$ is known [MNT00,MNT01]. As we see later, we make use of a bilinear map group system where we require the existence of an efficient isomorphism goint from $G_{2}$ to $G_{1}$. In symmetric setting, we have $G_{1}=G_{2}$. Known examples of $e(\cdot, \cdot)$ usually have $G_{1}, G_{2}$ to be the groups of Elliptic Curve or Hyperelliptic Curve points and $\tilde{G}$ to be a subgroup of a multiplicative group of finite field. Modified Weil pairing [BF01], Tate pairing [BKLS02,GHS02] are some of the practical examples of bilinear maps. 


\section{$2.4(n, t)$-MSE-DDH (The Multi-sequence of Exponents Diffie-Hellman Assumption)}

Our scheme's security relies on the hardness of a problem that we call the $(n, t)$ multi-sequence of exponents Diffie-Hellman problem (MSE-DDH). This problem falls under the general Diffie-Hellman exponent problem framework [BBG05]. Some of the problems that are similar to $(n, t)$-MSE-DDH, were considered in [DPP07,HLR10,DP08] and all of them fit the framework of general DiffieHellman exponent problem. [BBG05] provides an intractability bound for the general Diffie-Hellman exponent problem in the generic model [Sho97], where the underlying groups are equipped with pairings. Thus the generic complexity of $(n, t)$-MSE-DDH and the other similar problems mentioned in

[DPP07,HLR10,DP08] are covered by the analysis in [BBG05]. A proof to show the $(n, t)$-MSE-DDH problem as a particular instance of general Diffie-Hellman exponent problem is similar to the proof of [DP08], where it has been shown that the $(l, m, t)$-MSE-DDH $(l, m, t$ are integers) problem fit the framework of general Diffie-Hellman exponent problem.

Let $G_{1}, G_{2}, \tilde{G}$ be the three groups of the same prime order $p$, and let $e$ : $G_{1} \times G_{2} \rightarrow \tilde{G}$ be a non-degenerate and efficiently computable bilinear map. Let $g_{1}$ be a generator of $G_{1}$ and $g_{2}$ be a generator of $G_{2}$.

Let $n, t$ be two positive integers $(t \leq n)$. The $(n, t)$-multi-sequence of exponents Diffie-Hellman problem $((n, t)-\mathrm{MSE}-\mathrm{DDH})$ related to the group triplet $\left(G_{1}, G_{2}, \tilde{G}\right)$ is as follows:

- Input: Two polynomials $\theta_{1}, \theta_{2}$ as

$$
\theta_{1}(x)=\prod_{i=1}^{n}\left(x+a_{i}\right) \text { and } \theta_{2}(x)=\prod_{i=1}^{n-t+1}\left(x+b_{i}\right)
$$

where $a_{1}, \ldots, a_{n}$ and $b_{1}, \ldots, b_{n-t+1}$ are all distinct elements in $\mathbb{F}_{p}$. Thus degrees of $\theta_{1}, \theta_{2}$ are $n$ and $n-t+1$ respectively. We call $a_{1}, \ldots, a_{n}$ and $b_{1}, \ldots, b_{n-t+1}$ to be the negative roots of $\theta_{1}, \theta_{2}$ respectively. Beside polynomials $\theta_{1}, \theta_{2}$, the following sequences of exponentiations are also given as input,

- $\hat{g_{1}}:=\left[g_{1}, g_{1}^{\alpha},\left\{g_{1}^{\gamma^{i}}\right\}_{i=1}^{n+t-2},\left\{g_{1}^{\alpha \gamma^{i}}\right\}_{i=1}^{n+t}\right.$ and $\left.g_{1}^{k \alpha \theta_{1}(\gamma)}\right]$,

- $\hat{g_{2}}:=\left[g_{2}, g_{2}^{\alpha},\left\{g_{2}^{\gamma^{i}}\right\}_{i=1}^{n-t-1},\left\{g_{2}^{\alpha \gamma^{i}}\right\}_{i=1}^{n}\right.$ and $\left.g_{2}^{k \theta_{2}(\gamma)}\right]$,

- an element $T \in \tilde{G}$,

where $k, \alpha, \gamma \in \mathbb{F}_{p}^{*}$ and are not known.

- Output: a bit $b \in\{0,1\}$ as,

$$
b=\left\{\begin{array}{cc}
1 & \text { if } T=e\left(g_{1}, g_{2}\right)^{k \theta_{1}(\gamma)} \\
0 & \text { if } T \text { is a random element of } \tilde{G}
\end{array}\right.
$$

Thus the problem is to distinguish if $T$ is a random value or if it is equal to $e\left(g_{1}, g_{2}\right)^{k \theta_{1}(\gamma)}$. To be more precise, let us denote by real the event that $T=$ $e\left(g_{1}, g_{2}\right)^{k \theta_{1}(\gamma)}$, by random the event that $T$ is a random element from $\tilde{G}$ and by $\mathcal{I}\left(\theta_{1}, \theta_{2}, \hat{g_{1}}, \hat{g_{2}}, T\right)$ the input of the problem. Let $\lambda$ be the size of the underlying group order. We define the advantage of an algorithm $\mathcal{A}$ in solving $(n, t)$-MSEDDH problem as 


$$
\begin{gathered}
\operatorname{Adv}_{\mathcal{A}}^{(n, t)-\operatorname{MSE}-D D H}(\lambda)=\mid \operatorname{Pr}\left[\mathcal{A}\left(\mathcal{I}\left(\theta_{1}, \theta_{2},\left[g_{1}\right],\left[g_{2}\right], T\right)\right)=\right. \\
1 \mid \text { real }]-\operatorname{Pr}\left[\mathcal{A}\left(\mathcal{I}\left(\theta_{1}, \theta_{2},\left[g_{1}\right],\left[g_{2}\right], T\right)\right)=1 \mid \text { random }\right] \mid
\end{gathered}
$$

where the probability is taken over all the random coins consumed by $\mathcal{A}$.

\section{The new $(t, n)$-threshold PVSS scheme}

The earlier proposals for (publicly) verifiable secret sharing scheme mostly rely on the idea of interpolating a polynomial (see Appendix A) on the exponent of a generator of a group. A sketch of the idea can be given as follows:

- Fix a cyclic group $G$ of prime order $p$ and a generator $g \in G$.

- Choose a polynomial $f \in \mathbb{F}_{p}[x]$ of degree $t-1$, say $f(x)=a_{0}+a_{1} x+\cdots+$ $a_{t-1} x^{t-1}$.

- The polynomial is kept secret but a commitment to the polynomial is published by publicly distributing the coefficient of $f$ on the exponent of $g$. Shares (usually $f(i)$ 's for the $i$ th shareholder) are also published on the exponents of $g$.

- When $t$ or more participants come together, they can interpolate $f$ on the exponent of $g$, i.e., $g^{f(x)}$.

Our proposal, though works with polynomial interpolation, is based on a different approach. This idea is very prominent in threshold cryptography, e.g., broadcast encryption, threshold encryption, attribute based encryption etc. The approach for our scheme is inspired by the work of [DPP07,HLR10,DP08]. An overview of this idea can briefly be described as follows:

- Fix a cyclic group $G$ of prime order $p$ and a generator $g \in G$.

- Choose a polynomial $f \in \mathbb{F}_{p}[x]$ and publish it (unlike the earlier approach, $f$ is not kept secret).

- Instead what is kept secret is a value (say $\gamma \in \mathbb{F}_{p}$ ) where this polynomial would later be evaluated. Some public information is made available so that one can compute $g^{f(\gamma)}$.

Scheme: Now we describe a $(t, n)$-threshold publicly verifiable secret sharing scheme. A special property of this scheme is that the participants are initially issued secret keys such that for every new secret that the dealer wants to share, the participants can use the same secret keys to derive the respective shares of the secret in question. Let $\lambda$ be the underlying security parameter of the system.

- Initialization: This algorithm consists of two steps:

- Setting up public parameters: Generates a bilinear map group system $\left(p, G_{1}, G_{2}, \tilde{G}, e(\cdot, \cdot)\right)$. Let $\phi: G_{2} \rightarrow G_{1}$ be an efficiently computable group isomorphism. Also, two generators $g \in G_{1}$ and $h \in G_{2}$ are randomly selected as well as the secret values $\alpha, \gamma \in \mathbb{F}_{p}^{*}$. We assume that $p$ is significantly larger than $n$. The dealer then computes and publishes 


$$
\left(u=g^{\alpha \gamma}, h, h^{\alpha},\left\{h^{\gamma^{i}}\right\}_{i=1}^{n-t-1},\left\{h^{\alpha \gamma^{i}}\right\}_{i=1}^{n}\right)
$$

- User keys generation: There are $n$ participants $P_{1}, \ldots, P_{n}$ and each of them is given a pair of public key and secret key as: the dealer first randomly selects $n$ many distinct elements $a_{1}, \ldots, a_{n} \in \mathbb{F}_{p}^{*}$ and consider the following polynomial,

$$
f(x)=\prod_{i=1}^{n}\left(x+a_{i}\right)
$$

Then the $i$ th participant $P_{i}$ is given public key and secret key as

$$
\left(p k_{i}, s k_{i}\right)=\left(a_{i}, g^{\frac{1}{\gamma+a_{i}}}\right)
$$

Thus $\left\{a_{i}\right\}$ 's are known to all, i.e., $f$ is public. The Remark 1 below describes how the participants can verify the correctness of their respective secret keys. Also the dealer can publicly send the encrypted secret keys using any standard ElGamal like public key encryption scheme.

- Distribution: The dealer wishes to share a secret, which is an element in $\tilde{G}$. The secret is of the form $e(g, h)^{\alpha k}$, where $k$ is selected randomly from $\mathbb{F}_{p}^{*}$. The dealer then computes and publishes the following values:

- Share commitment element (SCE): This value binds the dealer's commitment to the secret and is given as,

$$
\mathrm{SCE}=u^{-k}=g^{-k \alpha \gamma}
$$

- Share deriving element (SDE): This value contains information about all the shares of the secret for which the dealer rendered his commitment. Participants will get their share by using the respective secret keys with $\mathrm{SDE}$. This value is given as,

$$
\mathrm{SDE}=h^{\alpha k f(\gamma)}
$$

The $i$ th participant gets his share $S_{i}$ by computing,

$$
S_{i}=e\left(g^{\frac{1}{\gamma+a_{i}}}, \mathrm{SDE}\right)
$$

- Verification: Any (external) verifier first computes and checks the following equality,

$$
e\left(\phi\left(h^{\alpha}\right), h^{\sum_{i=0}^{n-t-1} \gamma^{i}}\right)=e\left(\phi(h), h^{\sum_{i=0}^{n-t-1} \alpha \gamma^{i}}\right)
$$

Then it computes,

$$
\mathrm{SCE}^{\prime}=u^{-1} \text { and } \mathrm{SDE}^{\prime}=h^{\alpha f(\gamma)}
$$

One may note that $h^{\alpha f(\gamma)}$ can be computed using $\left\{h^{\alpha \gamma^{i}}\right\}_{i=1}^{n}$. The verifier then check the correctness by checking

$$
e\left(\mathrm{SCE}^{\prime}, \mathrm{SDE}\right)=e\left(\mathrm{SCE}, \mathrm{SDE}^{\prime}\right)
$$

One should also note that the share deriving element SDE is consistent with the share commitment element SCE if and only if there exists a scalar $k$ such that $\mathrm{SCE}=\left(\mathrm{SCE}^{\prime}\right)^{k}$ and $\mathrm{SDE}=\left(\mathrm{SDE}^{\prime}\right)^{k}$. If the verification fails, all participants exit the protocol.

- Reconstruction: Let $A$ be a qualified set of participants, i.e. it consists of at least $t$ many participants. Let the public-keys of the participants are $a_{r_{1}}, \ldots, a_{r_{s}}, s \geq t$. Together with their respective shares $e\left(g^{\frac{1}{\gamma+a_{r_{i}}}}, h^{\alpha k f(\gamma)}\right)$ 's, they reconstruct the secret as follows. They first compute 


$$
R_{1}=e(g, h)^{k \alpha f_{r_{1}, \ldots, r_{s}}(\gamma)}, \text { where } f_{r_{1}, \ldots, r_{s}}(\gamma)=\frac{f(\gamma)}{\prod_{i=1}^{s}\left(\gamma+a_{r_{i}}\right)}
$$

[The computation of $R_{1}$ is done recursively. A simple case is described here for convenience. With $e\left(g, h^{\alpha k f(\gamma)}\right)^{\frac{1}{\gamma+a_{r_{1}}}}$ and $e\left(g, h^{\alpha k f(\gamma)}\right)^{\frac{1}{\gamma+a_{r_{2}}}}$, the element $e\left(g, h^{\alpha k f(\gamma)}\right)^{\frac{1}{\left(\gamma+a_{r_{1}}\right)\left(\gamma+a_{r_{2}}\right)}}$ is derived as:

$$
\left(\frac{e\left(g, h^{\alpha k f(\gamma)}\right)^{\frac{1}{\gamma+a_{r_{1}}}}}{e\left(g, h^{\alpha k f(\gamma)}\right)^{\frac{1}{\gamma+a_{r_{2}}}}}\right)^{\frac{1}{\left(a_{r_{2}}-a_{r_{1}}\right)}}
$$

Thus, in order to compute $e\left(g, h^{\alpha k f(\gamma)}\right)^{\frac{1}{\left(\gamma+a_{r_{1}}\right)\left(\gamma+a_{r_{2}}\right)\left(\gamma+a_{r_{3}}\right)}}$, one can repeat the above technique twice: first with the inputs $e\left(g, h^{\alpha k f(\gamma)}\right)^{\frac{1}{\gamma+a_{r_{2}}}}$ and $e\left(g, h^{\alpha k f(\gamma)}\right)^{\frac{1}{\gamma+a_{r_{3}}}}$ (which will output $e\left(g, h^{\alpha k f(\gamma)}\right)^{\frac{1}{\left(\gamma+a_{r_{2}}\right)\left(\gamma+a_{r_{3}}\right)}}$ ) and secondly with the inputs $e\left(g, h^{\alpha k f(\gamma)}\right)^{\frac{1}{\left(\gamma+a_{r_{1}}\right)\left(\gamma+a_{r_{2}}\right)}}$ and $\left.e\left(g, h^{\alpha k f(\gamma)}\right)^{\frac{1}{\left(\gamma+a_{r_{2}}\right)\left(\gamma+a_{r_{3}}\right)}}\right]$ Next they compute,

$$
R_{2}=h^{\frac{1}{\gamma}\left(f_{r_{1}, \ldots, r_{s}}(\gamma)-f_{r_{1}, \ldots, r_{s}}(0)\right)}
$$

The computation of $R_{2}$ can successfully be carried out using $\left\{h^{\gamma^{i}}\right\}_{i=1}^{n-t-1}$ as degree of $\frac{1}{\gamma}\left(f_{r_{1}, \ldots, r_{s}}(\gamma)-f_{r_{1}, \ldots, r_{s}}(0)\right)=n-s-1$ and $n-s-1 \leq n-t-1$ as $(t \leq s)$. Now compute

$$
\begin{aligned}
e\left(\mathrm{SCE}, R_{2}\right) \cdot R_{1} & =e\left(g^{-k \alpha \gamma}, h^{\frac{1}{\gamma}\left(f_{r_{1}, \ldots, r_{s}}(\gamma)-f_{r_{1}, \ldots, r_{s}}(0)\right)}\right) \cdot e(g, h)^{k \alpha f_{r_{1}, \ldots, r_{s}}(\gamma)} \\
& =e(g, h)^{-k \alpha\left(f_{r_{1}, \ldots, r_{s}}(\gamma)-f_{r_{1}, \ldots, r_{s}}(0)\right)} \cdot e(g, h)^{k \alpha f_{r_{1}, \ldots, r_{s}}(\gamma)} \\
& =e(g, h)^{k \alpha f_{r_{1}, \ldots, r_{s}}(0)}
\end{aligned}
$$

Finally the secret is reconstructed by computing

$$
e(g, h)^{k \alpha}=\left(e(g, h)^{k \alpha f_{r_{1}}, \ldots, r_{s}(0)}\right)^{\frac{1}{f_{r_{1}, \ldots, r_{s}}(0)}}
$$

Remark 1. The $i$ th participant $P_{i}$, with its pair of keys

$$
\left(\mathrm{pk}_{i}, \mathrm{sk}_{i}\right)=\left(a_{i}, g^{\frac{1}{\gamma+a_{i}}}\right)
$$

can check the correctness of its secret key as follows. It first computes $h^{\alpha \gamma a_{i}}$ and checks if

$$
\begin{aligned}
e\left(\mathrm{sk}_{i}, h^{\alpha \gamma^{2}} \cdot h^{\alpha \gamma a_{i}}\right) & =e\left(g^{\frac{1}{\gamma+a_{i}}}, h^{\alpha \gamma^{2}} \cdot h^{\alpha \gamma a_{i}}\right) \\
& =e\left(g^{\frac{1}{\gamma+a_{i}}}, h^{\alpha \gamma\left(\gamma+a_{i}\right)}\right) \\
& =e\left(g^{\alpha \gamma}, h\right)=e(u, h)
\end{aligned}
$$

Remark 2. Our scheme couldn't provide a satisfactory answer to the following problem. During the reconstruction phase when a shareholder releases his share commitment value $e\left(g^{\frac{1}{\gamma+a_{i}}}, h^{\alpha k f(\gamma)}\right)$, there seems to be no obvious method to verify this value. One, not so interesting, wayout is to publish the hash digests of $e\left(g^{\frac{1}{\gamma+a_{i}}}, h^{\alpha k f(\gamma)}\right)^{\prime}$ 's, $(1 \leq i \leq n)$ during the distribution step of the scheme. But then this would mean that the correctness of the verification can only be carried out in the random oracle model. 
Remark 3. The Reconstruction algorithm of the scheme requires the computation of $R_{1}=e(g, h)^{k \alpha f_{r_{1}, \ldots, r_{s}}(\gamma)}$ given $\left\{a_{r_{i}}\right\}_{i=1}^{s}$ and $\left\{e\left(g^{\frac{1}{\gamma+a_{r_{i}}}}, h^{\alpha k f(\gamma)}\right)\right\}_{i=1}^{s}$. The recursive method (described in the scheme) takes time that is bounded by $\frac{(s-1) s}{2} \cdot\left(T_{p}+T_{\tilde{G}}\right)$, where $T_{p}$ is the total time of a subtraction and an inversion in $\mathbb{F}_{p}$ and $T_{\tilde{G}}$ the total time of a division and exponentiation in $\tilde{G}$. One may note that the computation of the elements $\left(\frac{e\left(g, h^{\alpha k f(\gamma)}\right)^{\frac{1}{\gamma+a_{r_{i}}}}}{e\left(g, h^{\alpha k f(\gamma)}\right)^{\frac{1}{\gamma+a_{r_{j}}}}}\right)^{\frac{1}{\left(a_{r_{j}}-a_{r_{i}}\right)}}$ 's is done by exponentiation (not by computing the high order roots) as $\left(a_{r_{j}}-a_{r_{i}}\right)$ is invertible modulo the order of the elements $\left(\frac{e\left(g, h^{\alpha k f(\gamma)}\right)^{\frac{1}{\gamma+a_{r_{i}}}}}{e\left(g, h^{\alpha k f(\gamma)}\right)^{\frac{1}{\gamma+a_{r_{j}}}}}\right)$ which is $p$.

\subsection{Security Analysis}

In this Section we present the security analysis of our scheme.

\section{$3.2 \quad$ Verifiability}

We describe that a dishonest dealer cannot cheat the shareholders without being detected in the verification.

Lemma 1. If the dealer passes the verification step, then all qualified subsets of honest shareholders will reconstruct the same secret that dealer had wished to share.

The dealer puts forward its commitment to the secret $e(g, h)^{\alpha k}$ by binding its essential value $k$ as part of the secret commitment element $\mathrm{SCE}=u^{-k}=g^{-k \alpha \gamma}$. Thus, the consistency of the pubilc parameters $\left(u=g^{\alpha \gamma}, h, h^{\alpha},\left\{h^{\gamma^{i}}\right\}_{i=1}^{n-t-1}\right.$, $\left.\left\{h^{\alpha \gamma^{i}}\right\}_{i=1}^{n}\right)$ and of the share deriving element $\mathrm{SDE}=h^{k \alpha f(\gamma)}$ with the secret commitment element SCE follows from the facts that,

$-e\left(\phi\left(h^{\alpha}\right), h^{\sum_{i=0}^{n-t-1} \gamma^{i}}\right)=e\left(\phi(h), h^{\sum_{i=0}^{n-t-1} \alpha \gamma^{i}}\right)$,

- $\operatorname{SDE}^{\prime}\left(=h^{\alpha f(\gamma)}\right)$ and $\mathrm{SCE}^{\prime}$ are obtained respectively from the dealer's publicly committed values $\left\{h^{\alpha \gamma^{i}}\right\}_{i=1}^{n}$ and $u=g^{\alpha \gamma}$,

- and the equality $e\left(\mathrm{SCE}^{\prime}, \mathrm{SDE}\right)=e\left(\mathrm{SCE}, \mathrm{SDE}^{\prime}\right)$ which essentially ensures that the scalar $k$ is same such that $\mathrm{SCE}=\left(\mathrm{SCE}^{\prime}\right)^{k}$ and $\mathrm{SDE}=\left(\mathrm{SDE}^{\prime}\right)^{k}$.

\subsection{Indistinguishability of Secrets (IND)}

In this section, we show that our $(t, n)$-threshold PVSS scheme is SA-IND secure, assuming that the $(n, t)$-MSE-DDH problem is hard to solve.

Theorem 1. Let $n, t(t \leq n)$ be two positive integers. For any PPT adversary $\mathcal{A}$ against the SA-IND security of our $(t, n)$-threshold PVSS scheme, there exists an algorithm $\mathcal{B}$ that distinguishes the two distributions of the $(n, t)-M S E-D D H$ problem, such that 


$$
\operatorname{Adv}_{\mathcal{A}}^{S A-I N D}(\lambda) \leq 2 \cdot \operatorname{Adv}_{\mathcal{B}}^{(n, t)-M S E-D D H}(\lambda)
$$

Proof: The security reduction is to show that if there is an adversary $(\mathcal{A})$ which can break our $(t, n)$-threshold PVSS then one obtains an algorithm to solve $(n, t)$-MDE-DDH. The heart of such an algorithm is a simulator $(\mathcal{B})$ which is constructed as follows. Given an instance of $(n, t)-\mathrm{MSE}-\mathrm{DDH}$ as input, the simulator plays the security game SA-IND with an adversary against $(t, n)$-threshold PVSS. The simulator sets up the $(t, n)$-threshold PVSS based on the $(n, t)$-MSE$\mathrm{DDH}$ instance. The simulator gives the public parameters to the adversary and continues the game by answering all queries made by the adversary. The queries include, public keys of $n$ participants and private keys of $t-1$ corrupted participants. In the process, it randomly chooses a bit $b$ and distributes the shares of the secret $T_{b}$ using the $(n, t)$-MSE-DDH instance provided as input. Finally, the adversary outputs a bit $b^{\prime}$. Based on the value of $b$ and $b^{\prime}$, the simulator decides whether the instance it received is real or random. Intuitively, if the adversary has an advantage in breaking the scheme, the simulator also has an advantage in distinguishing between real and random instances. This leads to an upper bound on the advantage of the adversary in terms of the advantage of the simulator in solving $(n, t)$-MSE-DDH.

- $(n, t)$-MSE-DDH Instance: The simulator, $\mathcal{B}$, receives an instance of $(n, t)$-MSE-DDH as described in Section 2.4. Thus $\mathcal{B}$ is given a bilinear map group system $\left(p, G_{1}, G_{2}, \tilde{G}, e(\cdot, \cdot)\right)$ where the size of $p$ is $\lambda$. We assume that $p$ is significantly larger than $n$. $\mathcal{B}$ is further given polynomials $\theta_{1}, \theta_{2} \in \mathbb{F}_{p}[x]$ of degrees $n$ and $n-t+1$ respectively as described in Section 2.4. The negative roots of $\theta_{1}$ and $\theta_{2}$ are denoted by $a_{1}, \ldots, a_{n}$ and $a_{n+t}, \ldots, a_{2 n}$ respectively. This instance also includes,

- $\hat{g_{1}}:=\left[g_{1}, g_{1}^{\alpha},\left\{g_{1}^{\gamma^{i}}\right\}_{i=1}^{n+t-2},\left\{g_{1}^{\alpha \gamma^{i}}\right\}_{i=1}^{n+t}\right.$ and $\left.g_{1}^{k \gamma \theta_{1}(\gamma)}\right]$,

- $\hat{g_{2}}:=\left[g_{2}, g_{2}^{\alpha},\left\{g_{2^{\gamma^{i}}}\right\}_{i=1}^{n-t-1},\left\{g_{2}^{\alpha \gamma^{i}}\right\}_{i=1}^{n}\right.$ and $\left.g_{2}^{k \theta_{2}(\gamma)}\right]$,

- an element $T \in \tilde{G}$.

- Initialization: $\mathcal{B}$ selects randomly $t-1$ elements $a_{n+1}, \ldots, a_{n+t-1} \in \mathbb{F}_{p}^{*}$ (different from the input $a_{i}$ 's) and construct a polynomial of degree $t-1$ as,

$$
\theta_{0}(x)=\prod_{i=n+1}^{n+t-1}\left(x+a_{i}\right)
$$

The public parameters are defined and published in the following manner.

- $g=g_{1}^{\theta_{1}(\gamma) \theta_{0}(\gamma)}$,

- $h=g_{2}$,

- $h^{\alpha},\left\{h^{\gamma^{i}}\right\}_{i=1}^{n-t-1},\left\{h^{\alpha \gamma^{i}}\right\}_{i=1}^{n}$,

- $u=g_{1}^{\alpha \gamma \theta_{1}(\gamma) \theta_{0}(\gamma)}=\left(g_{1}^{\theta_{1}(\gamma) \theta_{0}(\gamma)}\right)^{\alpha \gamma}=g^{\alpha \gamma}$.

One may note that $\mathcal{B}$ cannot compute $g$, as degree of $\theta_{1}(x) \theta_{0}(x)$ is $n+t-1$. As we see subsequently that the form of $g$ is required only for the security analysis and $\mathcal{B}$ doesn't have to publish it. Of course $\mathcal{B}$ can compute $u$ with $\left\{g_{1}^{\alpha \gamma^{i}}\right\}_{i=1}^{n+t}$. Thus to be precise the published parameters are,

$$
u, h, h^{\alpha},\left\{h^{\gamma^{i}}\right\}_{i=1}^{n-t-1},\left\{h^{\alpha \gamma^{i}}\right\}_{i=1}^{n}
$$


- User Keys Generation: There are $n$ participants and $t-1$ of them are assumed to be corrupted, i.e., $\mathcal{B}$ will issue respective public key and secret key pairs to $\mathcal{A}$ for $t-1$ corrupted participants and only public keys for the remaining $n-t+1$ participants.

- Corrupted participants: For $t-1$ corrupted participants

$\left(P_{w_{n+1}}, \ldots, P_{w_{n+t-1}}\right)$, the key pairs are issued to $\mathcal{A}$ as

$$
\left(\mathrm{pk}_{w_{i}}, \mathrm{sk}_{w_{i}}\right)=\left(a_{i}, g_{1}^{\frac{\theta_{1}(\gamma) \theta_{0}(\gamma)}{\gamma+a_{i}}}\right)=\left(a_{i}, g^{\frac{1}{\gamma+a_{i}}}\right), i=n+1 \text { to } n+t-1
$$

- Honest Participants: The remaining $n-t+1$ participants assigned their respective public keys from

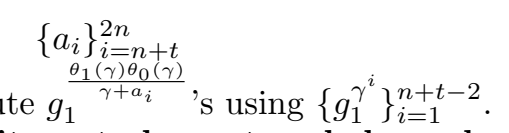

One may note that $\mathcal{B}$ can compute $g_{1}^{\frac{\theta_{1}(\gamma) \theta_{0}(\gamma)}{\gamma+a_{i}}}$ 's using $\left\{g_{1}^{\gamma^{i}}\right\}_{i=1}^{n+t-2}$

- Distribution of secret commitment element and share deriving element: $\mathcal{B}$ defines polynomial $f$, as described in the scheme, whose negative roots correspond to the public keys of all the participants. Thus,

$$
f(x)=\theta_{0}(x) \theta_{2}(x)
$$

$\mathcal{B}$ then proceed to select $T$ as the secret that it intends to share among the $n$ participants by publishing the secret commitment element and share deriving element as,

$$
(\mathrm{SCE}, \mathrm{SDE})=\left(g_{1}^{k \alpha \theta_{1}(\gamma)}, g_{2}^{k \theta_{2}(\gamma)}\right)
$$

One may note that, if we set $k^{\prime}=\frac{k}{\alpha \theta_{0}(\gamma)}$, then

$$
\begin{aligned}
\mathrm{SCE} & =g_{1}^{-k \gamma \theta_{1}(\gamma)} \\
& =g_{1}^{-k^{\prime} \alpha \gamma \theta_{0}(\gamma) \theta_{1}(\gamma)} \\
& =\left(g_{1}^{\theta_{1}(\gamma) \theta_{0}(\gamma)}\right)^{-k^{\prime} \alpha \gamma} \\
& =(g)^{-k^{\prime} \alpha \gamma}=u^{-k^{\prime}}
\end{aligned}
$$

and

$$
\begin{aligned}
\mathrm{SDE} & =g_{2}^{k \theta_{2}(\gamma)} \\
& =g_{2}^{\alpha k^{\prime} \theta_{0}(\gamma) \theta_{2}(\gamma)} \\
& =h^{\alpha k^{\prime} f(\gamma)}
\end{aligned}
$$

Further, if $T$ is real, then

$$
\begin{aligned}
T & =e\left(g_{1}, g_{2}\right)^{k \theta_{1}(\gamma)} \\
& =e\left(g_{1}, g_{2}\right)^{\alpha k^{\prime} \theta_{0}(\gamma) \theta_{1}(\gamma)} \\
& =e(g, h)^{\alpha k^{\prime}}
\end{aligned}
$$

Thus the secret $T$ is of required form as described in the scheme. With this, the simulator now randomly selects a bit $b \in\{0,1\}$ and sets $T_{b}=T$ and assigns a random value in the secret space $\tilde{G}$ to $T_{1-b}$. $\mathcal{A}$ is then issued 
(SCE, $\left.\mathrm{SDE}, T_{0}, T_{1}\right)$

- Guess: Finally $\mathcal{A}$ outputs its guess, a bit $b^{\prime}$ for $b$.

Based on the value of $b$ and $b^{\prime}, \mathcal{B}$ goes on to solve the $(n, t)$-MSE-DDH problem instance at hand as follows:

- if $b^{\prime}=b, \mathcal{B}$ answers 1 , meaning that $T=e\left(g_{1}, g_{2}\right)^{k \theta_{1}(\gamma)}$,

- otherwise, $\mathcal{B}$ answers 0 , meaning that $T$ is a random element of $\tilde{G}$.

Thus, the advantage of the algorithm $\mathcal{B}$ in solving the input $(n, t)$-MSE-DDH problem is

$$
\begin{aligned}
& \operatorname{Adv}_{\mathcal{B}}^{(n, t)-M S E-D D H}(\lambda) \\
& =\mid \operatorname{Pr}\left[\mathcal{B}\left(\mathcal{I}\left(\theta_{1}, \theta_{2},\left[g_{1}\right],\left[g_{2}\right], T\right)\right)=1 \mid \text { real }\right]-\operatorname{Pr}\left[\mathcal{B}\left(\mathcal{I}\left(\theta_{1}, \theta_{2},\left[g_{1}\right], \hat{g_{2}}, T\right)\right)=1 \mid \text { random }\right] \mid \\
& =\mid \operatorname{Pr}\left[b^{\prime}=b \mid \text { real }\right]-\operatorname{Pr}\left[b^{\prime}=b \mid \text { random }\right] \mid .
\end{aligned}
$$

In the above simulation, when the event real occurs, the simulator $\mathcal{B}$ poses as a real challenger for $\mathcal{A}$, i.e., the distribution of all the parameters during the simulation perfectly comply with the IND security game and therefore $\mid \operatorname{Pr}\left[b^{\prime}=\right.$ $b \mid$ real $]-\frac{1}{2} \mid=\frac{1}{2} \operatorname{Adv}_{\mathcal{A}}^{S A-I N D}(\lambda)$. Whereas, when the event random occurs, the distribution of the guess bit $b^{\prime}$ is completely independent of the distribution of the bit $b$ and thus $\operatorname{Pr}\left[b^{\prime}=b\right]$ is equal to $\frac{1}{2}$. Putting it altogether, we obtain

$$
\operatorname{Adv}_{\mathcal{A}}^{S A-I N D}(\lambda) \leq 2 \cdot \operatorname{Adv}_{\mathcal{B}}^{(n, t)-M S E-D D H}(\lambda)
$$

\section{Comparison}

We use $\mathcal{H V}$ and $\mathcal{P S}$ to denote the schemes proposed in [HV08] and this article respectively. In Table 1, we compare $\mathcal{P S}$ with $\mathcal{H V}$ in terms of exponentiations (in the underlying groups) and pairing computations. $\mathcal{H V}$ uses symmetric pairing and $\mathcal{P S}$ is based on asymmetric pairing. Thus the group exponents are computed in $G$ (see Section B) for $\mathcal{H V}$, and in $G_{1}, G_{2}$ for $\mathcal{P S}$ (see Section 3). A list of points to better understand the comparison table is given as follows:

$-n, t$ bears the usual meaning.

- For Reconstruction algorithm, comparison is done based on the number of operations required for $t$ shareholders to reconstruct the secret.

- $\mathcal{H} \mathcal{V}$ requires computation of $2 t$ pairings to verify the shares, released by the $t$ shareholders during the Reconstruction algorithm. But this number has not been counted in the comparison table as $\mathcal{P} \mathcal{S}$ does not satisfy this property. 
Table 1. Comparison Table

\begin{tabular}{llllc} 
Algorithms & Schemes & $\begin{array}{l}\text { Exponentiation } \\
\text { in } G, G_{1} \text { or } G_{2}\end{array}$ & $\begin{array}{l}\text { Exponentiation } \\
\text { in } \tilde{G}\end{array}$ & Pairing \\
\hline \multirow{2}{*}{ Setup } & $\mathcal{H} \mathcal{V}$ & $n$ & - & - \\
& $\mathcal{P S}$ & $3 n-t+1$ & - & - \\
\hline \multirow{2}{*}{ Distribution } & $\mathcal{H} \mathcal{V}$ & $n+t$ & - & - \\
& $\mathcal{P S}$ & 2 & - & $n$ \\
\hline \multirow{2}{*}{ Verification } & $\mathcal{H} \mathcal{P}$ & $n \cdot t$ & - & $2 n$ \\
& $\mathcal{P S}$ & $n$ & - & 4 \\
\hline \multirow{2}{*}{ Reconstruction } & $\mathcal{H} \mathcal{V}$ & $2 t$ & - & 1 \\
& $\mathcal{P S}$ & $n-t-1$ & $\approx \frac{(t-1) t}{2}$ & 1 \\
\hline
\end{tabular}

\section{Conclusion}

We have proposed in this paper a practical $(t, n)$-threshold PVSS scheme that achieves simultaneously:

- Efficient non-interactive public verification.

- Provable security for the public verifiability in the standard model.

Efficiency of the non-interactive public verification step of our scheme is optimal while comparing with the earlier proposal [HV08]. We provide formal security proof for our scheme, against indistinguishability of secrets (IND) attack model, based on the hardness of a problem that we call the $(n, t)$ - multi-sequence of exponents Diffie-Hellman problem (MSE-DDH). This problem falls under the general Diffie-Hellman exponent problem framework. [BBG05]. The security proof (for indistinguishability of secrets ) could handle only static adversaries. An interesting task would be to modify the scheme accordingly so that an adaptive adversary can be handled during the security analysis. The other challenging task is to provide the security proof under a more standard assumption.

\section{References}

[BBG05] Dan Boneh, Xavier Boyen, and Eu-Jin Goh. Hierarchical identity based encryption with constant size ciphertext. In EUROCRYPT, pages 440456, 2005.

[BF01] Dan Boneh and Matthew K. Franklin. Identity-based encryption from the weil pairing. In CRYPTO, pages 213-229, 2001.

[BKLS02] Paulo S. L. M. Barreto, Hae Yong Kim, Ben Lynn, and Michael Scott. Efficient algorithms for pairing-based cryptosystems. In CRYPTO, pages 354-368, 2002.

[Bla79] G. Blakley. Safeguarding cryptographic keys. AFIPS National Computer Conference, 48:313-317, 1979.

[BLS01] Dan Boneh, Ben Lynn, and Hovav Shacham. Short signatures from the weil pairing. In ASIACRYPT, pages 514-532, 2001. 
[BOGW88] Michael Ben-Or, Shafi Goldwasser, and Avi Wigderson. Completeness theorems for non-cryptographic fault-tolerant distributed computation (extended abstract). In STOC, pages 1-10, 1988.

[BR93] Mihir Bellare and Phillip Rogaway. Random oracles are practical: A paradigm for designing efficient protocols. In ACM Conference on Computer and Communications Security, pages 62-73, 1993.

[BW06] Xavier Boyen and Brent Waters. Anonymous hierarchical identity-based encryption (without random oracles). In CRYPTO, pages 290-307, 2006.

[CCD88] David Chaum, Claude Crépeau, and Ivan Damgård. Multiparty unconditionally secure protocols (extended abstract). In STOC, pages 11-19, 1988.

[CGMA85] Benny Chor, Shafi Goldwasser, Silvio Micali, and Baruch Awerbuch. Verifiable secret sharing and achieving simultaneity in the presence of faults (extended abstract). In FOCS, pages 383-395, 1985.

[DP08] Cécile Delerablée and David Pointcheval. Dynamic threshold public-key encryption. In CRYPTO, pages 317-334, 2008.

[DPP07] Cécile Delerablée, Pascal Paillier, and David Pointcheval. Fully collusion secure dynamic broadcast encryption with constant-size ciphertexts or decryption keys. In Pairing, pages 39-59, 2007.

[Fel87] Paul Feldman. A practical scheme for non-interactive verifiable secret sharing. Foundations of Computer Science, Annual IEEE Symposium on, 0:427-438, 1987.

[FO98] Eiichiro Fujisaki and Tatsuaki Okamoto. A practical and provably secure scheme for publicly verifiable secret sharing and its applications. In $E U$ ROCRYPT, pages 32-46, 1998.

[FS86] Amos Fiat and Adi Shamir. How to prove yourself: Practical solutions to identification and signature problems. In CRYPTO, pages 186-194, 1986.

[GHS02] Steven D. Galbraith, Keith Harrison, and David Soldera. Implementing the tate pairing. In ANTS, pages 324-337, 2002.

[GM84] Shafi Goldwasser and Silvio Micali. Probabilistic encryption. J. Comput. Syst. Sci., 28(2):270-299, 1984.

[HLR10] Javier Herranz, Fabien Laguillaumie, and Carla Ràfols. Constant size ciphertexts in threshold attribute-based encryption. In Public Key Cryptography, pages 19-34, 2010.

[HV08] Somayeh Heidarvand and Jorge L. Villar. Public verifiability from pairings in secret sharing schemes. In Selected Areas in Cryptography, pages 294308, 2008.

[Mic92] Silvio Micali. Fair public-key cryptosystems. In CRYPTO, pages 113-138, 1992.

[MNT00] Atsuko Miyaji, Masaki Nakabayashi, and Shunzo Takano. Characterization of Elliptic Curve Traces under FR-Reduction. In ICISC, pages 90-108, 2000.

[MNT01] Atsuko Miyaji, Masaki Nakabayashi, and Shunzo Takano. New Explicit Conditions of Elliptic Curve Traces for FR-Reduction. IEICE Transactions on Fundamentals, E84-A(5):1234-1243, 2001.

[Ped91] Torben P. Pedersen. Non-interactive and information-theoretic secure verifiable secret sharing. In CRYPTO, pages 129-140, 1991.

[Rev] Anonymous Review. Program committee of Indocrypt 2010.

[RV05] Alexandre Ruiz and Jorge Luis Villar. Publicly verfiable secret sharing from paillier's cryptosystem. In $W E W o R C$, pages 98-108, 2005. 
[Sch99] Berry Schoenmakers. A simple publicly verifiable secret sharing scheme and its application to electronic. In CRYPTO, pages 148-164, 1999.

[Sha79] A. Shamir. How to share a secret. Communications of the ACM, 22(11):612-613, 1979.

[Sho97] Victor Shoup. Lower bounds for discrete logarithms and related problems. In EUROCRYPT, pages 256-266, 1997.

[Sim88] Gustavus J. Simmons. How to (really) share a secret. In CRYPTO, pages 390-448, 1988.

[Sta96] Markus Stadler. Publicly verifiable secret sharing. In EUROCRYPT, pages 190-199, 1996.

[Sti92] Douglas R. Stinson. An explication of secret sharing schemes. Des. Codes Cryptography, 2(4):357-390, 1992.

\section{A The Lagrange Interpolation}

Here, we give a concrete description of the Lagrange interpolation. Let $P(x)=$ $\sum_{j=0}^{t-1} \alpha_{j} x^{j}$ be a polynomial over $\mathbb{F}_{p}$ with degree $t-1$ where $p$ is a prime, and $\left(x_{1}, P\left(x_{1}\right)\right), \ldots,\left(x_{t}, P\left(x_{t}\right)\right)$ be $t$ many distinct points over $P(x)$. Then, for given $\left(x_{1}, P\left(x_{1}\right)\right), \ldots,\left(x_{t}, P\left(x_{t}\right)\right)$ one can reconstruct $P(x)$ as

$$
P(x)=P\left(x_{1}\right) \lambda_{x_{1}}(x)+\cdots+P\left(x_{t}\right) \lambda_{x_{t}}(x)
$$

where for $1 \leq j \leq t$

$$
\lambda_{x_{j}}(x)=\frac{\left(x-x_{1}\right) \cdots\left(x-x_{j-1}\right)\left(x-x_{j+1}\right) \cdots\left(x-x_{t}\right)}{\left(x_{j}-x_{1}\right) \cdots\left(x_{j}-x_{j-1}\right)\left(x_{j}-x_{j+1}\right) \cdots\left(x_{j}-x_{t}\right)}
$$

\section{B Heidarvand and Villar's PVSS Scheme}

Here, we recall the PVSS scheme of [HV08]. A bilinear map group system $(p, G, \tilde{G}, e(\cdot, \cdot))$ is generatd where the bilinear map is $e: G \times G \rightarrow \tilde{G}$. The aim is to share efficiently a random value from $\tilde{G}$. The Dealer, $\mathcal{D}$, will achieve this by first randomly selecting two independent generators $g, h \in G, s \in \mathbb{F}_{p}$ and then distributing shares of the secret $e(h, h)^{s}$.

- Initialization: Participants $P_{i}$ 's generates their respective private keys $x_{i} \in R \mathbb{Z}_{p}^{*}$ and registers $y_{i}=h^{x_{i}}$ as their respective public keys.

- Distribution: The dealer wishes to distribute the secret among participants $P_{1}, \ldots, P_{n}$. The dealer picks a random polynomial $p$ of degree $t-1$ in $\mathbb{F}_{p}[x]$ :

$$
p(x)=\sum_{j=0}^{t-1} \alpha_{j} x^{j}
$$

and sets $s=\alpha_{0}$. The dealer keeps this polynomial secret but publishes the secret commitment values $C_{j}=g^{\alpha_{j}}, 0 \leq j \leq t-1$. The dealer also publishes the shares deriving values $Y_{i}=y_{i}^{P(i)}, 1 \leq i \leq n$, where $y_{i}$ 's are the public keys of the participants. 
- Verification: An external verifier can check the correctness of the shares as follows. For $i=1$ to $n$, it computes

$$
X_{i}=\prod_{j=0}^{t-1} C_{j}^{i^{j}}
$$

and checks if the following equalities holds.

$$
e\left(X_{i}, y_{i}\right)=e\left(g, Y_{i}\right)
$$

If the verification fails, all participants exit the protocol. Note that the verification step requires $2 n$ pairing computations.

- Reconstruction: Using its private key $x_{i}$, each participant find the share $S_{i}=h^{P(i)}$ from $Y_{i}$ by computing $S_{i}=Y_{i}^{x_{i}^{-1}}$. Then all participants pool their shares. All shares can be verified by other participants by checking the equation $e\left(S_{i}, y_{i}\right)=e\left(Y_{i}, h\right)$. After the verification, if there are at least $t$ correct shares, then for an arbitrary set A of $t$ participants who have pooled correct shares can get $h^{s}$ by Lagrange interpolation:

$$
\prod_{P_{i} \in A} S_{i}^{\lambda_{i}}=\prod_{P_{i} \in A}\left(h^{P(i)}\right)^{\lambda_{i}}=h^{\sum_{P_{i} \in A} \lambda_{i} P(i)}=h^{P(0)}=h^{s}
$$

where $\lambda_{i}=\prod_{P_{j} \in A \backslash\left\{P_{i}\right\}} \frac{j}{j-i}$ is a Lagrange coefficient. The secret will be recovered by computing $e\left(h^{s}, h\right)$.

\section{B.1 Security}

We state the security theorem for public verification of Heidarvand and Villar's PVSS Scheme [HV08].

Theorem 2. [HVO8] The PVSS scheme is publicly verfiable in the presence of an unbounded adversary.

\section{A Modification of Heidarvand and Villar's PVSS Scheme (Modified- $\mathcal{H} \mathcal{V}$ Scheme)}

In this Section we propose a modification to the verification algorithm of [HV08]. Thus we present only the new verification algorithm.

- Verification: An external verifier can check the correctness of the shares as follows. For $i=1$ to $n$, it computes

$$
X_{i}=\prod_{j=0}^{t-1} C_{j}^{i^{j}}
$$

and checks if the following equality holds,

$$
\prod_{i=1}^{n} e\left(X_{i}, y_{i}\right)=e\left(g, \prod_{i=1}^{n} Y_{i}\right)
$$

If the verification fails, all participants exit the protocol. Note that the verification step requires $n+1$ pairing computations. 


\section{C.1 $(n, t)$-RDHE (The Representation of $(n, t)$ Diffie-Hellman Exponent) Problem}

We relate the security of the public verifiability of Modified- $\mathcal{H V}$ to a variant of computational Diffie-Hellman problem. We call it, the $(n, t)$-RDHE (The Representation of ( $n, t)$ Diffie-Hellman Exponent) Problem.

Let $G$ be a multiplicative group with prime order $p$ and $n, t$ be two positive integers $(t \leq n)$. We assume that $p$ is significantly larger than $n$. The representation of $(n, t)$ Diffie-Hellman exponent problem related to the group $G$ is described as follows:

- Input: A degree $(t-1)$ polynomial in $\mathbb{F}_{p}[x]$,

$$
P(x)=\alpha_{0}+\alpha_{1} x+\ldots+\alpha_{t-1} x^{t-1}
$$

Beside $P(x)$, the tuple of exponents $\left[h, h^{d_{1}}, \ldots, h^{d_{n}}\right]$ is also given as input, where $h \in G$ and $d_{1}, \ldots, d_{n} \in \mathbb{F}_{p}$.

- Output: A tuple $\left(h^{d_{1}}\right)^{a_{1}}, \ldots,\left(h^{d_{n}}\right)^{a_{n}}$ such that,

$$
\left(a_{1}, \ldots, a_{n}\right) \neq(P(1), \ldots, P(n)) \text { but } h^{\sum_{i=1}^{n} d_{i} a_{i}}=h^{\sum_{i=1}^{n} d_{i} P(i)}
$$

where $a_{1}, \ldots, a_{n} \in \mathbb{F}_{p}$.

Let $\lambda$ be the size of the underlying group order. We define the advantage of an algorithm $\mathcal{A}$ in solving $(n, t)$-RDHE problem as

$$
\begin{gathered}
\operatorname{Pr}\left[\mathcal{A}\left(P(x), h, h^{d_{1}}, \ldots, h^{d_{n}}\right)=\left(h_{1}^{d} a_{n}^{a_{1}}, \ldots,\left(h_{n}^{d}\right)^{a_{n}}\right) \mid\left(a_{1}, \ldots, a_{n}\right) \neq\right. \\
\left.(P(1), \ldots, P(n)) \text { but } h^{\sum_{i=1}^{n} d_{i} a_{i}}=h^{\sum_{i=1}^{n} d_{i} P(i)}\right]
\end{gathered}
$$

where the probability is over the random choice of generator $h$ in $G$, the random choice of $P(x) \in \mathbb{F}_{p}[x]$, and the random bits consumed by $\mathcal{A}$.

Definition 2. We say that the $(\tau, \epsilon)-(n, t)-R D H E$ assumption holds on $G$ if no $\tau$-time algorithm has advantage at least $\epsilon$ in solving the $(n, t)$-RDHE problem on G.

\section{C.2 Security}

The following theorem states that if the dealer passes the verification, then all participants in the protocol must behave honestly or will be detected.

Theorem 3. Under the (n,t)-RDHE assumption, If Verifier accepts, then there exists unique polynomial $P(x)$ such that the encrypted share of participant $P_{i}$ is $Y_{i}=y_{i}^{P(i)}$ for $1 \leq i \leq n$.

Proof: The security reduction is to show that if there is a dishonest dealer $\mathcal{A}$ who can successfully cheat in the verification algorithm of the Modified- $\mathcal{H} \mathcal{V}$ scheme, then one obtains an algorithm $\mathcal{B}$ to efficiently solve $(n, t)$-RDHE problem. We describe the algorithm $\mathcal{B}$ as follows: 
$-\mathcal{B}$ is given an instance of the $(n, t)$-RDHE problem. This instance includes a polynomial $P(x)=\alpha_{0}+\alpha_{1} x+\ldots+\alpha_{t-1} x^{t-1}$, and a tuple $\left[h, h^{d_{1}}, \ldots, h^{d_{n}}\right]$ of elements from the group $G$ of order $p$.

- $\mathcal{B}$ selects randomly an element $g \in G$. It then fed $\mathcal{A}$ with $\left(P(x), g, h, h^{d_{1}}, \ldots, h^{d_{n}}\right)$ and asks $\mathcal{A}$ to setup the Modified- $\mathcal{H} \mathcal{V}$ scheme with $y_{i}=h^{d_{i}}$ as the public key of the $i$ th participant $P_{i}, 1 \leq i \leq n$.

- $\mathcal{A}$ then sets $e(h, h)^{\alpha_{0}}$ as the secret, where $\alpha_{0}=P(0)$.

- $\mathcal{A}$ then publishes the commitment values (for the polynomial $P(x)) C_{j}=$ $g^{\alpha_{j}}, 0 \leq j \leq t-1$. Next it selects $a_{1}, \ldots, a_{n}$ and publishes $Y_{i}=y_{i}^{a_{i}}, 1 \leq$ $i \leq n$. $\mathcal{A}$ then claims that $Y_{i}$ 's are the respective encrypted shares for the participants $P_{i}$ 's. Thus $\mathcal{A}$ can successfully cheats in the above claim (amounts to cheating in verification) if

$$
\left(a_{1}, \ldots, a_{n}\right) \neq(P(1), \ldots, P(n)) \text { but } \prod_{i=1}^{n} e\left(X_{i}, y_{i}\right)=e\left(g, \prod_{i=1}^{n} Y_{i}\right) .
$$

- Finally $\mathcal{B}$ outputs $\left(Y_{1}, \ldots, Y_{n}\right)$ as the solution to the given $(n, t)$-RDHE problem instance.

This completes the description of $\mathcal{B}$. Equation (1) implies

$$
\left(a_{1}, \ldots, a_{n}\right) \neq(P(1), \ldots, P(n)) \text { but } h^{\sum_{i=1}^{n} d_{i} a_{i}}=h^{\sum_{i=1}^{n} d_{i} P(i)}
$$

as $\prod_{i=1}^{n} e\left(X_{i}, y_{i}\right)=e\left(g, \prod_{i=1}^{n} Y_{i}\right)=>\prod_{i=1}^{n} e\left(g^{P(i)}, h^{d_{i}}\right)=e\left(g, \prod_{i=1}^{n} y_{i}^{a_{i}}\right)=>$ $\prod_{i=1}^{n} e\left(g, h^{d_{i} \cdot P(i)}\right)=e\left(g, \prod_{i=1}^{n} h^{d_{i} \cdot a_{i}}\right)=>e\left(g, h^{\sum_{i=1}^{n} d_{i} \cdot P(i)}\right)=e\left(g, h^{\sum_{i=1}^{n} d_{i} \cdot a_{i}}\right)$ $=>h^{\sum_{i=1}^{n} d_{i} P(i)}=h^{\sum_{i=1}^{n} d_{i} a_{i}}$. Thus if the dealer $\mathcal{A}$ successfully cheats in the verification algorithm then its advantage translates to the advantage of $\mathcal{B}$ in solving the given $(n, t)$-RDHE problem instance. 